\title{
Piaceri di diverso genere. Donne e alcol: risposte non più disattendibili
}

\author{
Anna Paola Lacatena*, Paola Monopoli^, Vincenza Ariano ${ }^{\circ}$
}

\section{SUMMARY}

- Can we really believe that women's use of psychotropic substances is as limited with respect to men as national data, and others, would have us believe?

A study using the administration of MAST tests on the youth population of Taranto, conducted over the last 12 months by the Street Unit "Metroland", in collaboration with the Department of Pathological Addiction (Dipartimento delle Dipendenze Patologiche) of ASL Taranto, seems toundermine this notion.

From here, careful consideration towards reviewing crave-blocking methods would suggest the taking charge along with provision of care by the Addiction Services.

So it does not seem superfluous to restate that all patients are not the same, perhaps beginning with their gender.

Keywords: Women, Alcohol, Addiction Services, Shelter, Treatment.

Parole chiave: Donne, Alcol, Ser.D., Accoglienza, Trattamento.

\section{Introduzione}

Da operatori Ser.D. dovremmo chiederci se i dati che risultano dal nostro quotidiano operato rispecchiano davvero la realtà del consumo di sostanze psicotrope.

Dovremmo chiederci se quell'ormai consolidata differenza tra presenze maschili e presenze femminili, afferenti ai nostri Servizi, rifletta davvero un così significativo scarto tra consumatori uomini e consumatrici donne.

Pur rappresentando i Ser.D. la realtà di riferimento per la cura della dipendenza, gli stessi sono altrettanto significativamente in grado di fornire indicazioni in merito al consumo?

In estrema sintesi, possiamo davvero credere che il consumo di sostanze sia ad oggi ancora quasi esclusivamente una faccenda da uomini solo perché il dato a disposizione dei Servizi pende drasticamente verso questo verso?

Nella Relazione sugli interventi nel 2015 in materia di alcol e problemi correlati, trasmessa al Parlamento I'11 marzo 2016, il rapporto $M / F$ è pari a 3,7 sia per gli utenti totali che per gli utenti distinti per tipologia (nuovi e già in carico o rientrati); a livello regionale questa maggiore presenza maschile risulta più evidente al Centro-Sud.

Secondo i dati ISTAT, nel corso del 2014, il 63\% degli italiani di 11 anni e più (pari a 34 milioni e 319 mila persone) ha consumato almeno una bevanda alcolica, con prevalenza notevolmente maggiore tra i maschi $(76,6 \%)$ rispetto alle femmine $(50,2 \%)$.

\footnotetext{
* Dirigente Sociologa clo Dipartimento Dipendenze Patologiche ASL Taranto.

^Sociologa c/o Cooperativa "La Vela", Taranto.

-Direttore f.f. Dipartimento Dipendenze Patologiche ASL Taranto, Taranto.
}

Il $22,1 \%$ dei consumatori (12 milioni circa di persone) beve quotidianamente $(33,8 \%$ tra i maschi e $11,1 \%$ tra le femmine).

Nel 2014 si osserva ancora un lieve calo rispetto all'anno precedente dei consumatori giornalieri (nel 2013 rappresentavano il $22,7 \%$ e nel 2014 il 22,1\%) mentre continua ad essere in aumento il consumo di alcol occasionale e al di fuori dei pasti (binge drinking): nel 2013 erano il 25,8\% e nel 2014 il 26,9\%. II consumo fuori pasto è soprattutto diffuso tra i giovani (18-24 anni) e i giovani adulti (25-44), che lo adottano spesso nell'ambito di occasioni e contesti legati al divertimento e alla socializzazione.

Continua in modo preoccupante la crescita negli ultimi dieci anni del consumo fuori pasto tra le femmine, passato dal 14,9\% del 2005 al 16,5\% del 2014.

Tale crescita sembra essersi verificata in tutte le fasce di età, mentre il dato complessivo dei consumi fuori pasto tra i maschi risulta in lieve diminuzione (37,3\% nel 2005, 36,1\% nel 2014). Il fenomeno del binge drinking desta particolare allarme, con gravi rischi per la salute e la sicurezza non solo del singolo bevitore ma anche dell'intera società.

Nel 2014, il 10,0\% degli uomini e il 2,5\% delle donne di età superiore a 11 anni hanno dichiarato di aver consumato 6 o più bicchieri di bevande alcoliche in un'unica occasione almeno una volta negli ultimi 12 mesi.

Le percentuali di binge drinker sia di sesso maschile che femminile aumentano progressivamente nel secondo decennio di vita e raggiungono i valori massimi tra i 18-24enni ( $M=21,0 \%$; $\mathrm{F}=7,6 \%$ ); oltre questa fascia di età le percentuali diminuiscono nuovamente per raggiungere i valori minimi nell'età anziana $(\mathrm{M}=2,1 \% ; \mathrm{F}=0,3 \%)$.

La percentuale di binge drinker di sesso maschile è statisticamente superiore al sesso femminile in ogni classe di età ad eccezione degli adolescenti (11-15 anni), ossia quella fascia di popo- 
lazione per la quale la percentuale dovrebbe essere zero a causa del divieto per legge della vendita e somministrazione di bevande alcoliche al di sotto della maggiore età.

Preoccupante anche il consumo di alcol tra i cosiddetti "giovani anziani", persone di età compresa tra 65 e 74 anni.

Sono 2.700 .000 i consumatori ultra sessantacinquenni a rischio per patologie e problematiche alcol-correlate.

Dall'analisi del tipo di bevande consumate si conferma la tendenza già registrata negli ultimi dieci anni che vede una progressiva riduzione della quota di consumatori che bevono solo vino e birra, soprattutto fra i più giovani e le donne e un aumento della quota di chi consuma, oltre a vino e birra, anche aperitivi, amari e superalcolici, aumento che si registra nei giovani e i giovanissimi ma in misura percentuale maggiore negli adulti oltre i 44 anni e gli anziani.

Tra i giovani di 18-24 anni è in crescita il consumo di altri alcolici (aperitivi alcolici, alcolpops) oltre a vino e birra.

Per tutte le altre classi di età si osserva, tra gli uomini, una diminuzione sia il numero di quanti consumano solo vino e birra sia la quota di chi beve anche altri alcolici come aperitivi, amari e superalcolici; tra le donne è stabile la quota di chi beve anche altri alcolici ed è in diminuzione il numero di coloro che bevono solo vino e birra.

In ogni caso complessivamente tra le bevande consumate nel 2014 il vino resta al primo posto seguito dalla birra e quindi al terzo posto altri alcolici (aperitivi, amari e superalcolici).

(www.salute.gov.it/portale/news/p3_2_1_1_1.jsp?lingua=italiano\&menu=notizie $\& p=d a / m i n i s t e r o \& i d=2496$ )

\section{Come funziona (e come finisce per non funziona- re più) il sistema della gratificazione}

Probabilmente nessuna esperienza ha assunto nei secoli la capacità di dividere e contrapporre idee e percezioni quanto il piacere.

Il piacere, in estrema sintesi, sarebbe un sentimento o un'esperienza che induce l'essere umano in uno stato di benessere, in una condizione positiva.

Anche un gesto semplice e banale può essere espressione del piacere, purché determini contentezza nell'individuo.

Lo stesso agisce a livello psicologico con evidenti ricadute fisiche: pertanto siamo in grado di distinguere ciò che e bello e gratificante, da ogni cosa che provoca dolore e sofferenza.

Non a caso si tende a ripetere esperienze piacevoli, piuttosto che esperienze noiose e insignificanti, ma quando la ricerca del piacere diventa ossessiva, I'uomo finisce per diventarne dipendente.

Tutte le società hanno cercato di imporre un confine tra il piacere e la sua degenerazione, al fine di limitarne la caduta nel vizio, per usare un'espressione datata, o nella dipendenza patologica, preferendo un linguaggio più scientifico.

In verità, le moderne tecniche d'indagine sul funzionamento del nostro cervello ci dicono che molti comportamenti che consideriamo virtuosi (ad es. meditare o fare beneficenza), attivano lo stesso circuito neurale su cui agiscono, le sostanze stupefacenti e l'alcol e che i confini tra il socialmente accettabile e il moralmente deplorevole non sono poi così netti, chiamando in causa fattori cari alle neuroscienze ma anche e soprattutto aspetti culturali, morali e giuridici che danno forma al nostro essere e alla nostra identità all'interno della società (Linden, 2012).

Non meno dirimenti sono i fattori soggettivi, strettamente connessi alla storia e all'immaginario personale (Abraham, 2002).
Com'è funziona il meccanismo del piacere?

Chi spinge il pulsante, azionando il processo?

Per dare una risposta a questa domanda è opportuno osservare cosa succede nel cervello a proposito dei circuiti del piacere. Negli anni '50, i neurologi James Olds e Peter Milner, con degli esperimenti sui ratti, studiarono e analizzarono le strutture neurologiche coinvolte nel sistema della gratificazione.

Il piacere ha radici evoluzionistiche molto profonde, esistono, infatti, circuiti del piacere anche nel mondo non strettamente umano.

I batteri ingeriti dagli animali (serpenti, vermi, ecc.) nel corso dei pasti attivano i meccanismi di rilascio della dopamina e dunque del piacere.

Lo stimolo del piacere esisterebbe per incentivarci a sopravvivere (mangiare, bere, accoppiarsi, ecc.).

Con l'evoluzione, I'essere umano ha attivato meccanismi non naturali per innescare il piacere, ad esempio con I'allenamento intensivo, capace di produrre nel corpo molecole simili a quelle di cannabinoidi (endocannabinoidi).

A questo processo di induzione artificiale non si è sottratto neppure il mondo animale (vedi erba gatta vera, bacche, radici, frutta fermentata, ecc.).

Inoltre, nel corso dei secoli, il nostro cervello si è ingrandito progressivamente, in particolare nella parte frontale, responsabile del ragionamento e dei processi cognitivi e sociali, dando luogo a sempre più complesse connessioni.

Il centro del piacere è una struttura situata nel diencefalo (parte profonda del cervello), in grado di produrre sensazioni gratificanti, regolate da una particolare sostanza denominata dopamina.

Si tratta di un neurotrasmettitore, la cosiddetta "molecola del piacere", ovvero un segnale biochimico che consente ai neuroni di comunicare tra loro.

Essa viene liberata da un'altra struttura cerebrale, ossia I'area ventrale tegumentale (VTA).

Dai neuroni del VTA vi sono collegamenti con alcune regioni del centro del piacere, tra cui il nucleo accumbens e la corteccia prefrontale.

A sensazioni di appagamento e di piacere corrisponde un aumento della concentrazione della dopamina in quelle aree, essendo la stessa alla base dei meccanismi di regolazione della ricompensa e dell'omeostasi edonica.

Ogni qual volta mettiamo in atto qualcosa che ci piace, ci gratifica o ci rende felici, l'area ventrale tegumentale rilascia il neurotrasmettitore nel centro del piacere.

Quando assumiamo sostanze stupefacenti esse vanno ad agire proprio sul VTA, alterandone il corretto funzionamento nel provocare, essendo peraltro facilmente danneggiabile, un aumento innaturale della dopamina.

I sistemi di regolazione e limitazione della stessa, infatti, vengono bloccati dalle sostanze assunte, facendola aumentare esponenzialmente nel nucleo accumbens e nella corteccia prefrontale. Non essendoci più limite, la dipendenza trova terreno fertile. Il centro del piacere, poi, si abitua a quantità di dopamina sempre più consistenti, alle quali corrisponde un attaccamento allo stimolo gratificante sempre più pressante, e una sua assunzione sempre più elevata e frequente con un piacere che non è e non potrebbe più essere nemmeno paragonabile a quello provato agli esordi dell'assunzione o dell'iniziale messa in atto di quel determinato comportamento.

Per provare a semplificare, immaginiamo di essere molto affamati: se al primo boccone ci sottoponessimo alla scansione del nostro cervello, vedremmo una forte attivazione del centro del piacere. 
Continuando a mangiare, probabilmente, ci renderemmo conto che l'ultimo boccone, per quanto ancora gradito e apprezzabile, non avrà più lo stesso gusto del primo né segnerà la stessa gratificazione.

Di fatto il grado di attivazione del medial forebrain pleasure circuit decresce nel tempo, pur tenendo traccia viva della percezione soggettiva del piacere.

A tal proposito è bene precisare che l'illiceità di una sostanza non costituisce un deterrente rispetto a quello che è a tutti gli effetti un fenomeno neurobiologico.

Le sostanze che non attivano quest'area, come l'Lsd o la mescalina, non danno assuefazione; tutte le altre, siano esse naturali, semisintetiche o sintetiche, evidentemente sì.

Il sistema della gratificazione di una persona è come una detonazione anticipata dall'accensione di una miccia.

$\mathrm{Si}$ accende velocemente, altrettanto subitaneamente brucia e porta al piacere.

Non riesce a darsi una sorta di gradualità e di persistenza, non si manifesta lentamente né può dirsi durevole a lungo.

Nel tempo, l'impossibilità di riprodurre analogo piacere a quello derivato dalle prime assunzioni e l'alterazione del normale processo della gratificazione, spesso, sconfinano in veri e propri danni tra i quali e, quasi paradossalmente più determinati da sostanze che non inducono alla dipendenza (vedi cannabis), le sindromi depressive.

Nelle persone che hanno sviluppato dipendenze patologiche, queste ultime sembrano presentare tre caratteristiche ricorrenti. In primis, apatia, abulia e carenza di gratificazioni e di stimoli, capaci di condurre il cervello all'incapacità di identificare il piacere o di interessarsi a ciò che compone una normale vita quotidiana.

Si tratta di condizioni in cui si manifesta un atteggiamento fortemente distonico rispetto allo stato dell'umore. Inoltre, le persone coinvolte sembrano mal sopportare anche situazioni di depressione lievi in quanto l'asticella di riferimento del cervello non è più quella normale essendo l'euforia determinata dalla sostanza stessa.

Come a dire che la condizione di malessere è avvertita immediatamente sotto lo stare bene e non sotto I'asticella della sufficienza.

La seconda caratteristica ricorrente prevede che la depressione non sia neanche "completa", alternandosi in questi soggetti momenti di abulia e disinteresse ad altri di grande iperattività e smania di fare, di vivere, di sentire.

Questa latente e non sempre riconosciuta forma di insoddisfazione finisce per minare le relazioni e i legami affettivi, rendendo tutto molto caduco e passeggero.

L'ultimo tratto è il carattere misto delle depressioni.

"Spesso le persone con storie di uso di sostanze, presentano tratti di personalità particolari con temperamento reattivo, irritabile, vulnerabile. Dopo l'uso di sostanze l'ansia tende ad aumentare con una scarsa capacità nella persona di tollerarla. Vissuta con rabbia, urgenza, irrequietezza, angoscia sino a portare la persona nell'urgenza, nella confusione, nella necessità improrogabile di trovare vie d'uscita. Lo "stato misto dell'umore" induce la persona a cercare soluzioni per tenere a bada l'ansia e una di queste è proprio l'uso di sostanze che in questo caso vengono cambiate. II soggetto volge lo sguardo verso sostanze che non ha usato in passato e che percepisce come più accettabili socialmente e innocue, con un viraggio dalla ricerca di effetti stimolanti a effetti narcotici o comunque tranquillizzanti. Questo tipo di approccio non può che peggiorare nel tempo il quadro della depressione» (Piacere, anedonia, e depressione, di Matteo Pacini, consultabile in www.psi- chiatriaedipendenze.it/sintomi-disturbi/depressione-edistimia/piacere-anedonia-e-depressione/).

Il progressivo calo dell'umore, delle energie e della capacità di tollerare frustrazioni e stimoli spiacevoli è una diretta conseguenza anche dell'abuso di tranquillanti in persone (soprattutto donne) che non hanno alle spalle carriere tossicomaniche ma che hanno fatto ricorso a questi farmaci per controllare panico, ansia, insonnia, ecc.

Il trattamento di questi quadri non è semplice e diretto, perché di solito quando si studiano farmaci antidepressivi per valutarne I'efficacia, quanti hanno fatto uso di sostanze e alcol sono esclusi dal campionamento.

Il numero di persone che presentano questi disagi, però, è in costante crescita senza che vi sia per contro una rinnovata capacità di formulare risposte in grado di soddisfare le relative aspettative.

Frequentemente, poi, si incorre nella miopia di credere che l'uso di sostanze o il particolare comportamento scomparirà se l'umore migliorerà.

Di fatto, la dipendenza come malattia non può essere curata solo guardando alla depressione come origine e centro del disagio stesso, questo significherebbe non solo ritardare la diagnosi ma in alcuni casi sfociare nell'antiterapeutico dell'invalidazione della cura e del peggioramento della condizione iniziale. Tutto ciò è valido al maschile almeno quanto al femminile.

\section{La ricerca}

La conoscenza e l'informazione relativamente alle sostanze psicotrope legali o illegali si intersecano frequentemente con il tema della prevenzione consegnandoci, lì dove il rigore scientifico non venga sovrastato dalla spinta moralistica, all'idea delI'altissima diffusione dell'uso e della più circoscritta questione della dipendenza vera e propria.

Per quanto quest'ultima rappresenti un problema di grandissima serietà, non può essere vista come la modalità più diffusa di rapporto con le sostanze.

Da questo punto di vista si tratta, dunque, di costruire un percorso di conoscenza ad un livello più ampio, di comunità.

Affrontare la sfida sul terreno socio-culturale e della puntuale conoscenza è, forse, la sola reale possibilità di incidere sui comportamenti a rischio che non coinvolgono in maniera esclusiva le frange più giovani di popolazione.

Non solo effetti negativi e distruttivi con tutto l'allarmismo di cui siamo stati capaci per decenni ma conoscenza dei rischi reali soprattutto quando ad essere più nocive sono proprio le droghe legali.

Per non rimanere chiusi nella cura della cronicità o per non ridurre l'intervento alla burocratizzazione afflittiva di alcune normative relative al settore o all'intervento una tantum all'interno di istituti scolastici, nell'ottica di una prevenzione universale, il Dipartimento Dipendenze Patologiche ASL ha suggerito alla locale Amministrazione comunale di includere negli interventi relativi alle aree delle dipendenze (ridotta e scarsamente finanziata, come non è difficile immaginare) l'avvio di un progetto di intervento sul territorio, operato da giovani formati dagli operatori del DDP, in grado di incontrare e stabilire un contatto con la gente.

Deterritorializzazione del Servizio specialistico, dunque, contro il rischio della disinformazione, della collusione con la negazione del problema, della rimozione difensiva, della prevenzione come optional e non come diritto. 
Non un'iniziativa di strada rivolta a chi è già dentro il problema ma l'opportunità di sapere, di trovare indicazioni, notizie, informazioni.

Una sorta di avamposto del Ser.D. cittadino con la non secondaria finalità di tenere alta l'attenzione su queste problematiche, avvicinando il Servizio alla gente che, con buona probabilità, anche a seguito di un malevolo approccio all'idea stessa di qualcuno che guarda al dipendente patologico come un malato e non come un "vizioso", percepisce il luogo deputato alla cura in chiave negativa, stigmatizzante, periferica e ghettizzante.

L'Unità mobile "Metroland", progetto in collaborazione tra la Direzione Servizi Sociali del Comune di Taranto, Dipartimento Dipendenze Patologiche ASL TA e Cooperativa "La Vela" in attuazione dei Piani Sociali di Zona 2014-2016, prende il suo nome dal romanzo d'esordio dello scrittore Julian Barnes.

È noto come nella vita londinese la metropolitana (The Tube) rivesta un ruolo fondamentale.

Si tratta, a tutti gli effetti, della più antica del mondo, seconda per dimensioni solo a quella di Shanghai. Dodici sono le sue linee, tra cui la Metropolitan (colore viola), dove c'è la stazione che dà il titolo al romanzo: Metroland.

Di fatto una sottile striscia di terra del tutto sprovvista di unità geografica e progettuale come ideale terrain vague.

Il progetto, di durata annuale con la non celata aspettativa possa trovare continuità nel tempo, è stato avviato nel mese di aprile 2015, concludendosi nel maggio 2016.

\section{Metodologia "Metroland"}

Le uscite dell'Unità Mobile "Metroland" sono state calendarizzate e programmate dall'equipe previa mappatura dei luoghi e degli eventi destinati alla popolazione giovanile del Comune di Taranto.

I luoghi in cui "Metroland" ha operato sono stati: strada/piazza/giardini, concerti musicali, eventi musicali e culturali aperti al pubblico, iniziative ed eventi organizzati da esercenti di locali da ballo e non del territorio.

Alcuni incontri sono stati effettuati, con gli operatori del DDP ASL TA presso il Centro di Accoglienza per Minori Immigrati (c/o Chiesa "Corpus Domini", quartiere Paolo VI, Taranto), ad altri hanno partecipato testimoni privilegiati debitamente formati.

In estrema sintesi, l'attività si è orientata in termini di:

- Interventi di prossimità.

- Distribuzione di materiale informativo: le brochure distribuite sono state realizzate a cura del Ministero della Salute, dell'Osservatorio nazionale ACOL CNESPS, della Società Italiana Alcologia e della Onlus Donne in rete, e consegnate dal Ser.D. (Servizio Dipendenze) ASL di Taranto all'équipe dell'Unità Mobile Metroland.

NPS Italia (Network Persone Sieropositive) ha messo a disposizione a titolo gratuito profilattici e materiale relativo alle malattie sessualmente trasmissibili.

- Creazione di uno spazio virtuale L'équipe ha realizzato una pagina dedicata all'Unità Mobile Metroland sul social Network Facebook allo scopo di:

- pubblicizzare il servizio;

- agganciare soggetti che più facilmente in rete chiederebbero consigli o racconterebbero la loro storia;

- diffondere il maggior numero di informazioni sui rischi correlati all'uso e all'abuso di sostanze
Durante le uscite è stato proiettato il cortometraggio "la Dipendenza" della durata di 8 minuti circa, in cui non compaiono volti di ragazzi ma si fa ricorso all'uso di un linguaggio non verbale per descrivere metaforicamente i rischi legati all'uso/abuso di sostanze (dal lavoro già svolto dal DDP ASL TA DVD "Pro e Contro mano").

Il video si chiude con le indicazioni relative ai numeri telefonici del Ser.D. (Servizio Dipendenze) ASL di Taranto, del Alcol Istituto Superiore di Sanità Ministero della Salute, del link di Epicentro, portale dell'Epidemiologia per la Sanità Pubblica a cura del Centro Nazionale di Epidemiologia, Sorveglianza e Promozione della Salute

- Realizzazione e diffusione video: nel mese di Dicembre è stato realizzato e postato un breve video che invita alla guida sicura. Gli operatori del progetto Unita Mobile "Metroland" hanno realizzato nel mese di febbraio un cortometraggio postato sulla pagina facebook Unita Mobile con I'intento di estendere e rendere visibile, riconoscibile e fruibile il servizio e altresì catturare l'attenzione della fetta di popolazione più giovane e social.

- Realizzazione Evento "In-Dependent Art" del 29 aprile 2016 presso l'Auchan di Taranto

L'evento è stato pensato e organizzato dalla Società Cooperativa Sociale "La Vela"a conclusione del progetto del Servizio dell'Unità Mobile di strada "Metroland" con la supervisione degli operatori DDP ASL TA.

Lo stesso si è tenuto presso le gallerie Auchan.

Mauro Tatulli (Property Manager della Galleria Auchan di Taranto) oltre a mettere a disposizione l'area della galleria e della piazzetta interna adiacente alla zona di ristorazione ha offerto a tutti gli studenti convenuti un coupon per la consumazione di un panino e una bibita.

L'intento dell'iniziativa era quello di premiare il dipinto che meglio rappresentasse l'argomento delle dipendenze patologiche. Al lavoro ritenuto più espressivo dalla giuria, composta dai rappresentanti istituzionali dei Servizi Sociali del Comune di Taranto e del DDP Ser.D. ASL TA è stato consegnato un buono acquisto del valore di $€ 50,00$.

A seguire gli studenti del Liceo Artistico "Calò" (Grottaglie - TA)e dell'ISS "Liside" di Taranto sono stati impegnati in due momenti concomitanti:

- un estemporanea di pittura realizzata dagli stessi studenti che, muniti dei propri cavalletti posizionati lungo la gallerie del centro commerciale hanno rappresentato artisticamente le dipendenze patologiche o comportamentali;

- la partecipazione ad uno dei quattro Workshop tematici, sulle dipendenze tenuti da testimonial di associazioni di auto mutuo aiuto e comunità terapeutiche.

L'andamento del servizio è stato monitorato con i seguenti strumenti:

- Diario di Bordo in cartaceo, aggiornato dopo ogni uscita. Nel documento è stato riporta l'andamento di ogni uscita del pulmino rispetto all'affluenza dei contatti, alla durata dell'uscita, al numero degli operatori presenti ed eventuali altre osservazioni;

- Database in cui sono stati registrati il numero dei contatti distinti per genere e età.

Per la registrazione di questi dati è stato utilizzato un registro cartaceo mentre per l'elaborazione si è fatto ricorso al programma Excel di Windows 97;

- Schede di rilevazione del materiale distribuito in cui è stato specificato il numero dei contatti avuti, dei pieghevoli distribuiti e degli alcool test somministrati. 


\section{I materiali "Metroland"}

La metodologia di intervento è stata centrata su:

- formazione iniziale di tutto il personale impiegato nel Progetto a cura degli operatori del Dipartimento Dipendenze Patologiche ASL TA (medici, psicologi/psicoterapeuti, sociologi, assistenti sociali) sulle tematiche inerenti gli obiettivi dell'Unità Mobile;

- avvicinamento alle tematiche attraverso il confronto con testimoni privilegiati e portatori $d^{\prime}$ interesse (Club Alcolisti in Trattamento, Alcolisti Anonimi, Giocatori Anonimi, NPS ossia Network Persone Sieropositive);

- gli operatori "Metroland" hanno puntato sull'approccio relazionale di tipo pro-attivo e motivazionale al fine di favorire I'avvicinamento di giovani e meno giovani, stimolando lo scambio domanda/risposta.

Nel corso dell'attività dell'Unità mobile "Metroland", gli operatori hanno distribuito materiale informativo redatto dal Dipartimento Dipendenze Patologiche ASL TA e dall'Istituto Superiore di Sanità (Emanuele Scafato- Roma) nella cui ultima pagina sono stati inseriti dei test o questionari:

- CPGI (Canadian Problem Gambling Index), questionario di autovalutazione short form;

- MAST (Michigan Alcoholism Screening Test), short form;

- Test di Fagerström, test per la valutazione del grado di dipendenza dalla nicotina

La metodologia adottata è di tipo integrata in due fasi concomitanti: una di tipo qualitativo attraverso le interviste non strutturate (o colloqui informali) caratterizzate dalla mancanza di una scaletta o di un elenco di domande, ma sfruttando gli incontri e le conversazioni ordinarie; I'altra di tipo quantitativo attraverso la somministrazione di test.

I dati raccolti sono stati codificati in modo da poter essere trattati con strumenti informatici, classificati e conteggiati sia in valori assoluti che in termini percentuali, in modo da ottenere una descrizione complessiva. Per la somministrazione dei test si è ritenuto utile procedere con un campionamento non probabilistico.

Nella versione originaria, il questionario MAST (Michigan Alcoholism Screening Test), somministrato o autosomministrato, con i suoi 25 item, sonda il consumo di alcol e le sue complicanze.

Quello utilizzato dagli operatori dell'Unità di Strada è la versione Brief Mast (questionario autocompilato di 10 items).

I singoli item vengono valutati con punteggi differenti.

Largamente utilizzato, il test ha il vantaggio di essere agile e sufficientemente attendibile.

Evidentemente, i risultati dei Test sono da leggersi all'interno di una indagine conoscitiva realizzata su un campione che non è numericamente rappresentativo della popolazione giovane della città di Taranto. Somministrati per strada, percentuali e numeri vanno considerati spunti di riflessioni cui sarebbe utile abbinare ulteriori approfondimenti.

\section{L'Alcol e la norma disattesa}

L'Organizzazione Mondiale della Sanità (OMS) classifica l'alcol fra le droghe.

In Europa si ha il più elevato consumo alcolico al mondo.

Il consumo per abitante sembra essere il doppio rispetto alla media mondiale.
L'alcol è, infatti, il terzo fattore di rischio per i decessi e per le invalidità in Europa, e il principale fattore di rischio per la salute dei giovani (http://alcol.dronet.org/sostanza/oms.html).

Nel 1960 l'OMS cercò di inquadrare in modo razionale I'uso inadeguato dell'alcol e definì due condizioni principali: I'abitudine al bere e la tossicomania.

Nella prima è preponderante non la necessità ma il desiderio di assumere la sostanza per avvertire gli effetti piacevoli e il senso di benessere.

Appare assente o scarsa la tendenza ad aumentare le dosi.

È possibile il manifestarsi di una dipendenza psichica ma non ancora fisica, con effetti negativi solo per l'individuo.

Nella tossicomania, I'intossicazione è definita cronica e nociva non solo per il soggetto ma per la società.

È presente desiderio compulsivo dell'alcol e il bisogno di procurarselo con ogni mezzo, la tolleranza con la necessità di aumentare le dosi assunte, alla dipendenza psichica si accompagna anche quella fisica, con l'inevitabilità delle crisi di astinenza.

È sempre più condivisa dalla comunità scientifica la convinzione che i fattori psicologici possono avere un ruolo causale importante nell'alcol dipendenza solo se associati ad alcuni aspetti culturali, geografici, familiari, genetici.

Nel rapporto Epidemiologia e Monitoraggio alcol correlato in Italia e nelle Regioni (Scafato et al., 2016), I'ONA- (Osservatorio Nazionale Alcol), il CNESPS (Centro Nazionale di Epidemiologia Sorveglianza e Promozione della Salute) e I'ufficio di statistica dell'ISS (Istituto Superiore della Sanità) hanno elaborato i dati dell'indagine multiscopo annuale sulle famigli italiane dell'ISTAT e hanno messo in evidenza la valutazione del rischio alcolcorrelato nella popolazione, con particolare attenzione alle categorie più vulnerabili: giovani, donne e anziani.

"Il carico di malattie legate al consumo dannoso di alcol è notevole in molte parti del mondo sia in termini di morbilità che di mortalità. II consumo di alcol produce conseguenze dannose per la salute e conseguenze sociali per il bevitore, tra cui un aumento del rischio di una serie di tumori, dell'ictus e della cirrosi epatica. L'esposizione del feto all'alcol aumenta il rischio di difetti alla nascita e deficit cognitivi. L'alcol infine contribuisce alla morte e all'invalidità a causa degli incidenti, di lesioni, aggressioni, violenza, omicidi e suicidi [...]. Il consumo di alcol ha infine conseguenze sociali dovute alla perdita di produttività lavorativa a causa dell'assenteismo e della mortalità prematura, anche a causa delle lesioni e della morte dei non bevitori» (Scafato et al., 2016, p. 9).

Guardare ai dati prodotti dalla ricerca condotta dagli operatori dell'Unità Mobile "Metroland" del DDP ASL TA in materia di uso di alcol, in particolare tra i più giovani, significa che la normativa vigente è costantemente disattesa.

E questa è già una questione che meriterebbe la massima attenzione e una revisione, con buona probabilità, non della stessa legge ma della sua corretta osservanza.

L'art. 689 del Codice Penale vieta la somministrazione di alcol ai minori di anni 16 e a persone inferme di mente, punendo l'esercente che si sottrae al dettame normativo con l'arresto sino ad un anno.

Con I'art. 52, comma 2 lett. b., del D.Lgs. n. 274 del 2000, I'arresto è stato sostituito con la pena pecuniaria da 516 a 2582 euro o con la permanenza domiciliare da 15 a 45 giorni ovvero con la pena del lavoro di pubblica utilità da 20 giorni a 6 mesi, con la sospensione, in ogni caso, dell'esercizio dell'attività in caso di condanna già alla prima violazione (art.35 del codice penale). Il decreto legge Balduzzi, n. 158 del 2012, ha introdotto il divieto di vendita fino ai 18 anni prevedendo sanzioni amministrative e pecuniarie. 
Modificando la Legge 125 del 2001 in diversi punti, il decreto convertito in legge 189 del 2012, recita:

"Chiunque vende bevande alcoliche ha l'obbligo di chiedere all'acquirente, all'atto dell'acquisto, l'esibizione di un documento d'identità, tranne nei casi in cui la maggiore età dell'acquirente sia manifesta».

Inoltre, si legge: "Salvo che il fatto non costituisca reato, si applica la sanzione amministrativa pecuniaria da 250 a 1000 euro a chiunque vende bevande alcoliche ai minori di anni diciotto. Se il fatto è commesso più di una volta si applica la sanzione amministrativa pecuniaria da 500 a 2000 euro con la sospensione dell'attività per tre mesì.

Graf. 1 - Maschi intervistati (451 soggetti corrispondenti al 62\% del campione generale)

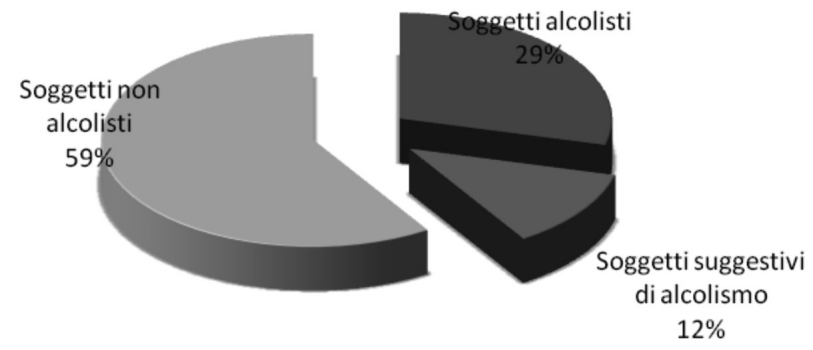

Il decreto aggiunge all'art. 689 c.p. il comma attraverso il quale è fatto divieto di somministrare ai minori di anni 16 anche attraverso distributori automatici.

\section{I dati "Metroland"}

Il campione totale di ragazzi contattati è risultato composto da 726 soggetti, 451 maschi (62\%) e 275 femmine (38\%) prevalentemente di età compresa tra gli 11 e i 25 anni.

Di seguito si riportano i dati elaborati con statistica descrittiva.

Graf. 2 - Femmine intervistate (275 femmine corrispondenti al 38\% del campione generale)

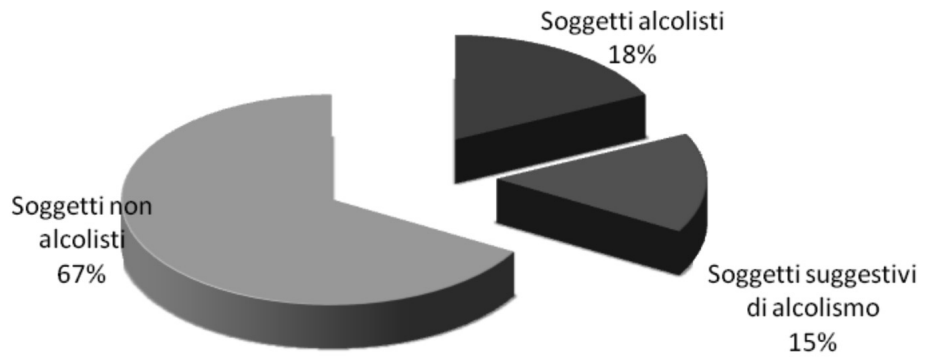

L'équipe dell'Unità Mobile ha effettuato un monitoraggio per ogni singola risposta suddivisa per genere che si rimanda di seguito.

Tab. 1 - Maschi Intervistati (451 soggetti-62\% del campione generale)

\begin{tabular}{|c|c|c|}
\hline Domande & SI & NO \\
\hline Pensi di bere normalmente? & $81 \%$ & $19 \%$ \\
\hline I tuoi amici o parenti pensano che bevi normalmente? & $73 \%$ & $27 \%$ \\
\hline Sei mai stato a una riunione di anonimi alcolisti? & $3 \%$ & $97 \%$ \\
\hline Hai mai perso un amico o una amica a causa del bere? & $11 \%$ & $89 \%$ \\
\hline Ti sei mai trovato in difficoltà sul lavoro a causa del bere? & $4 \%$ & $96 \%$ \\
\hline $\begin{array}{l}\text { Hai mai trascurato i tuoi obblighi, la tua famiglia o il tuo lavoro per due o più giorni di seguito } \\
\text { perché avevi bevuto? }\end{array}$ & $6 \%$ & $94 \%$ \\
\hline $\begin{array}{l}\text { Hai mai sofferto di Delirium Tremens, di brividi scuotenti o udito voci e visto cose che non esistevano } \\
\text { dopo una forte bevuta? }\end{array}$ & $11 \%$ & $89 \%$ \\
\hline Hai mai chiesto aiuto a qualcuno per la situazione creata dal bere? & $14 \%$ & $86 \%$ \\
\hline Sei mai stato in ospedale per aver bevuto? & $4 \%$ & $96 \%$ \\
\hline Sei mai stato arrestato per aver guidato in stato di ebbrezza o per aver guidato dopo aver bevuto? & $2 \%$ & $98 \%$ \\
\hline
\end{tabular}

Tab. 2 - Femmine Intervistate (275 soggetti - 38\% del campione generale)

\begin{tabular}{|c|c|c|}
\hline Domande & $S I$ & $\mathrm{NO}$ \\
\hline Pensi di bere normalmente? & $74 \%$ & $26 \%$ \\
\hline I tuoi amici o parenti pensano che bevi normalmente? & $69 \%$ & $31 \%$ \\
\hline Sei mai stato a una riunione di anonimi alcolisti? & $1 \%$ & $99 \%$ \\
\hline Hai mai perso un amico o una amica a causa del bere? & $8 \%$ & $92 \%$ \\
\hline Ti sei mai trovato in difficoltà sul lavoro a causa del bere? & $4 \%$ & $96 \%$ \\
\hline $\begin{array}{l}\text { Hai mai trascurato i tuoi obblighi, la tua famiglia o il tuo lavoro per due o più giorni di seguito } \\
\text { perché avevi bevuto? }\end{array}$ & $5 \%$ & $95 \%$ \\
\hline $\begin{array}{l}\text { Hai mai sofferto di Delirium Tremens, di brividi scuotenti o udito voci e visto cose che non esistevano } \\
\text { dopo una forte bevuta? }\end{array}$ & $7 \%$ & $93 \%$ \\
\hline Hai mai chiesto aiuto a qualcuno per la situazione creata dal bere? & $10 \%$ & $90 \%$ \\
\hline Sei mai stato in ospedale per aver bevuto? & $1 \%$ & $99 \%$ \\
\hline Sei mai stato arrestato per aver guidato in stato di ebbrezza o per aver guidato dopo aver bevuto? & 0 & $100 \%$ \\
\hline
\end{tabular}


Allo scopo di monitorare il comportamento dei minorenni circa I'uso delle sostanze alcoliche, dal campione di cui sopra è stato disaggregata la fascia di età compresa tra i 14 e i 16 anni.

Graf. 3 - Maschi intervistati 14/16 anni (108 soggetti corrispondenti al $62 \%$ del campione generale)

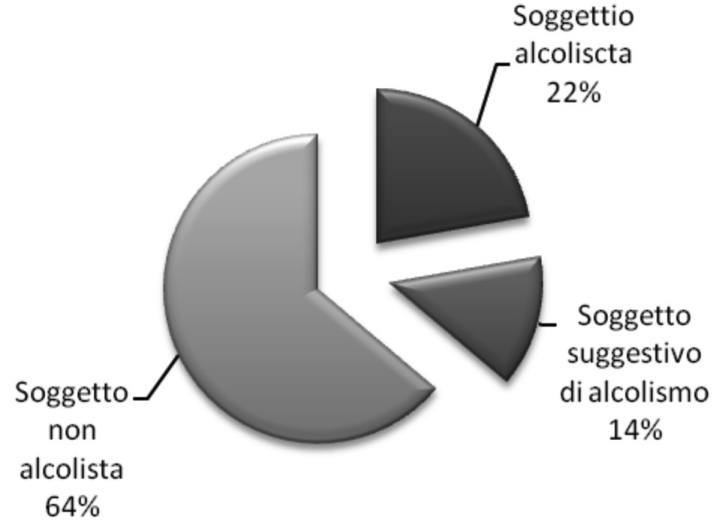

Il campione è risultato così composto: 174 soggetti, di cui 108 maschi $(62 \%)$ e 66 femmine (38\%).

Graf. 4 - Femmine intervistate 14/16 anni (66 femmine corrispondenti al $38 \%$ del campione generale)

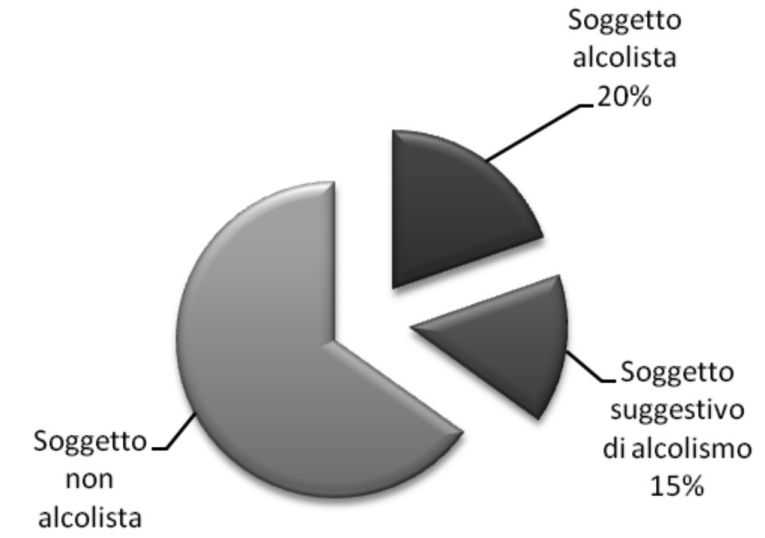

Di seguito si riportano in tabella a doppia entrata le risposte rilevate dai test auto-valutativi effettuati dalla fascia di popolazione giovane 14-16 anni.

Tab. 3 - Maschi Intervistati 14/16 anni (108 soggetti corrispondenti al 62\% del campione generale)

\begin{tabular}{|c|c|c|}
\hline Domande & SI & NO \\
\hline Pensi di bere normalmente? & $77 \%$ & $23 \%$ \\
\hline I tuoi amici o parenti pensano che bevi normalmente? & $72 \%$ & $28 \%$ \\
\hline Sei mai stato a una riunione di anonimi alcolisti? & $4 \%$ & $96 \%$ \\
\hline Hai mai perso un amico o una amica a causa del bere? & $6 \%$ & $94 \%$ \\
\hline Ti sei mai trovato in difficoltà sul lavoro a causa del bere? & $3 \%$ & $97 \%$ \\
\hline $\begin{array}{l}\text { Hai mai trascurato i tuoi obblighi, la tua famiglia o il tuo lavoro per due o più giorni di seguito } \\
\text { perché avevi bevuto? }\end{array}$ & $4 \%$ & $96 \%$ \\
\hline $\begin{array}{l}\text { Hai mai sofferto di Delirium Tremens, di brividi scuotenti o udito voci e visto cose che non esistevano } \\
\text { dopo una forte bevuta? }\end{array}$ & $13 \%$ & $87 \%$ \\
\hline Hai mai chiesto aiuto a qualcuno per la situazione creata dal bere? & $14 \%$ & $86 \%$ \\
\hline Sei mai stato in ospedale per aver bevuto? & $5 \%$ & $95 \%$ \\
\hline Sei mai stato arrestato per aver guidato in stato di ebbrezza o per aver guidato dopo aver bevuto? & $2 \%$ & $98 \%$ \\
\hline
\end{tabular}

Tab. 4 - Femmine Intervistate 14/16 anni (66 femmine corrispondenti al 38\% del campione generale)

\begin{tabular}{|c|c|c|}
\hline Domande & $S I$ & NO \\
\hline Pensi di bere normalmente? & $77 \%$ & $23 \%$ \\
\hline I tuoi amici o parenti pensano che bevi normalmente? & $63 \%$ & $37 \%$ \\
\hline Sei mai stato a una riunione di anonimi alcolisti? & $0 \%$ & $100 \%$ \\
\hline Hai mai perso un amico o una amica a causa del bere? & $10 \%$ & $90 \%$ \\
\hline Ti sei mai trovato in difficoltà sul lavoro a causa del bere? & $2 \%$ & $98 \%$ \\
\hline $\begin{array}{l}\text { Hai mai trascurato i tuoi obblighi, la tua famiglia o il tuo lavoro per due o più giorni di seguito } \\
\text { perché avevi bevuto? }\end{array}$ & $2 \%$ & $98 \%$ \\
\hline $\begin{array}{l}\text { Hai mai sofferto di Delirium Tremens, di brividi scuotenti o udito voci e visto cose che non esistevano } \\
\text { dopo una forte bevuta? }\end{array}$ & $8 \%$ & $92 \%$ \\
\hline Hai mai chiesto aiuto a qualcuno per la situazione creata dal bere? & $10 \%$ & $90 \%$ \\
\hline Sei mai stato in ospedale per aver bevuto? & $0 \%$ & $100 \%$ \\
\hline Sei mai stato arrestato per aver guidato in stato di ebbrezza o per aver guidato dopo aver bevuto? & $0 \%$ & $100 \%$ \\
\hline
\end{tabular}

In occasione del Concerto del $\mathrm{I}^{\circ}$ maggio tarantino I'Unità Mobile ha effettuato un monitoraggio esclusivo dei dati allo scopo di confrontarli con un campione analogo per distribuzione di gene- re ed età, raccolto durante l'uscita del 28 dicembre 2015 in una delle piazze più note e frequentate della città di Taranto (P.zza Maria Immacolata). 
Durante il "Concertone" sono stati contattati 125 soggetti: 64 maschi e 61 femmine di età compresa tra i 14-57. Nell'uscita del 28 dicembre sono stati contattati 121 soggetti: 63 maschi e 58 femmine di età compresa tra i 14-57 anni.

Di seguito si riportano in tabella le risposte rilevate dai test autovalutativi, somministrati nei due eventi succitati, e successivamente confrontate per genere.

Tab. 5 - Differenze intervistati maschi (Concerto $1^{\circ}$ maggio 2016 e 28 dicembre 2015)

\begin{tabular}{lcc}
\hline Maschi & Concerto 1 maggio & 28 dicembre 2015 \\
\hline Soggetto alcolista & $41 \%$ & $18 \%$ \\
Soggetto suggestivo & $12,5 \%$ & $19 \%$ \\
$\quad$ di alcolismo & $47 \%$ & $63 \%$ \\
\hline
\end{tabular}

Tab. 6 - Differenze intervistati femmine (Concerto $1^{\circ}$ maggio 2016 e 28 dicembre 2015)

\begin{tabular}{lcc}
\hline Femmine & Concerto 1 maggio & 28 dicembre 2015 \\
\hline $\begin{array}{l}\text { Soggetto alcolista } \\
\text { Soggetto suggestivo }\end{array}$ & $31 \%$ & $12 \%$ \\
$\quad$ di alcolismo & v.a. & $22 \%$ \\
Soggetto non alcolista & $69 \%$ & $66 \%$ \\
\hline
\end{tabular}

La somministrazione del test MAST (Michigan Alcoholism Screening Test) ha messo in evidenza una certa resistenza da parte dei ragazzi intervistati, che quasi sempre hanno espresso la loro condizione di sobrietà, salvo poi disegnare, attraverso le risposte al test, riscontri differenti.

Il monitoraggio effettuato sulla popolazione giovane tarantina dall'équipe dell'Unità Mobile risulta in linea con i dati rilevati dall'Osservatorio Epidemiologico della Regione Puglia del 2015. Nella fattispecie, il rapporto riferisce che il 57,8\% degli studenti di scuola secondaria ha l'abitudine di bere, nonché un "tendenziale progressivo incremento con l'aumentare dell'età".

Il dato che più di ogni altro colpisce la nostra attenzione è quello relativo al campione di età compresa tra i 14-16 anni.

I maschi soggetti all'alcol sono risultati il $22 \%$ e suggestivi all'alcol il 14\%; mentre le femmine risultate soggette all'alcol sono state il $20 \%$, e suggestive all'alcol il $15 \%$.

Inoltre, dalla lettura delle risposte fornite dal campione 14-16, il $13 \%$ dei maschi e l' $8 \%$ delle femmine afferma di aver sofferto di Delirium Tremens che, come anticipato in premessa si manifesta nei soggetti affetti da alcolismo cronico.

A tal proposito, è altamente probabile che i ragazzi abbiano associato l'esperienza personale di intossicazione da alcol, in seguito a una ubriacatura, al Delirium Tremens.

Prende corpo l'ipotesi, dunque, che anche fra i giovani tarantini (maschi e femmine) sia ampiamente diffuso il consumo di alcol e in maniera preoccupante il fenomeno del Binge Drinking.

\section{Donne, consumo e dipendenza}

Dagli ultimi dati pubblicati dall'Osservatorio Europeo sulle Droghe ( $d$ 'ora in poi OEDT) si conferma nel vecchio continente il protagonismo del genere maschile nell'ambito del consumo di stupefacenti.
Tale predominanza sarebbe rinvenibile in merito a tutte le droghe e a tutti gli stili di consumo, sebbene le disuguaglianze di genere siano difformi da paese a paese e da sostanza a sostanza; stessa prevalenza sarebbe registrabile nel numero di pazienti in trattamento presso i Servizi e le strutture di recupero, così come maggiormente rappresentato sarebbe il genere maschile tra i decessi per overdose (Cipolla, 2013).

Sembrerebbe, però, che in quelle realtà nazionali dove l'uguaglianza tra i sessi in merito alle forme di lavoro produttivo e riproduttivo sia più garantita, si registri un assottigliamento delle differenze di genere nel consumo di sostanze psicoattive.

Davvero dobbiamo credere che il numero delle donne che utilizzano droghe sia esiguo così come i dati ufficiali intendono suggerire?

E soprattutto, non è possibile parlare anche per le donne di consumi ricreazionali, restando limitatamente nell'alveo della dipendenza patologica?

In maniera generale, ci sarebbe da chiedersi in che modo la dimensione del genere declina i consumi di sostanze psicoattive.

Alcuni commentatori (Formica, Giuliodoro, 2009), infatti, ritengono sottostimati i numeri relativi al consumo femminile, guardando a questo aspetto come l'area più consistente del sommerso.

La stigmatizzazione legata al consumatore di sostanze, infatti, è da sempre più accentuata quando a consumare è la donna.

Tale fattore innegabilmente ha inciso sul consumo ma anche sulla cautela della donna a rivelarne lo stesso.

La "protezione" assicurata dall'uomo consumatore con cui, spesso, la donna ha condiviso lo stile di vita, la disconferma sociale ma più ancora la paura di conseguenze e ricadute in termini di gestione dei figli hanno consegnato il genere femminile all'ombra.

Se per anni gli studi relativi al consumo di sostanze hanno trascurato, per ragioni dettate dall'inconsistenza numerica, le donne, più di recente si assiste ad un crescente interesse verso I'universo femminile con rilevazioni statistiche che minano l'idea del consumo a quasi completo appannaggio degli uomini (Wallace et al., 2003; EMCDDA, 2006).

La normalizzazione del consumo, la facilità di reperimento della sostanza, la sua contiguità e disponibilità, la più diffusa accettazione sociale (accomodating), l'adattamento culturale rispetto alle droghe illegali, il tasso crescente di "sperimentatori", il tasso di "utilizzatori", l'attribuzione di significati differenti rispetto al passato, le ragioni dell'uso da distribuire in ordine al ricreativo, strumentale e performativo hanno indotto ad una rivisitazione di alcune convinzioni (Parker e Aldrige, 1998; Parker et al., 2002). L'ipotesi di Zuckerman (1983) di una maggiore inclinazione maschile al rischio e della teoria del sensation seeking necessita di una riflessione più articolata alla luce di dati più recenti.

Da una ricerca condotta da Cipolla e Martoni nel 2009 su 5233 frequentatori di locali notturni dai 15 anni in su, i consumatori di alcol (coloro che bevono nel weekend, più volte durante la settimana o tutti i giorni) risultano sorprendentemente distribuiti in maniera quasi equa tra uomini e donne $(70,1 \% \mathrm{M}$ e $62,4 \% \mathrm{~F})$. Circa una ragazza su due beve regolarmente durante il fine settimana.

Il fumo di tabacco quotidiano risulta per il 36\% ad appannaggio degli intervistati di sesso maschile e per il $34,8 \%$ per quelli di sesso femminile.

Hanno sperimentato la cannabis il $51,2 \%$ dei maschi a fronte del $38,8 \%$ delle femmine; la cocaina il $30 \%$ dei maschi e il $15 \%$ delle donne; I'ecstasy rispettivamente il $20 \%$ e il $10 \%$. 
Per le donne si tratterebbe di un consumo ricreazionale da condividere con altre persone sia in termini di consumo che di approvvigionamento (Dionigi, Pavarin, 2010), senza dimenticare l'uso terapeutico.

Due appaiono le questioni su cui riflettere a proposito di consumo al femminile.

Le differenze relative ai numeri del consumo sembrano assottigliarsi tra uomini e donne a proposito di utilizzo sporadico, tornando a segnare gap importanti per ciò che attiene a quello più sistematico e patologico.

Le eccezioni suggerite dall'OEDT in Europa riguardano prevalentemente le sostanze eccitanti (cocaina ed ecstasy), proponendosi in quelle realtà più evolute per ciò che attiene alla parità dei sessi.

Di fatto la tipologia sarebbe divisa tra un profilo di donna dipendente con resistenze ad avvicinarsi ai Servizi pubblici e della cura in generale (vedi Comunità Terapeutiche) per evitare lo stigma e possibili conseguenze, dovendo corrispondere a responsabilità precise in seno alla famiglia e dall'altra la giovane emancipata che non sembrerebbe avere un rapporto problematico con la sostanza ma più occasionale e circoscritte al loisor.

Per contro, la donna afferente ai Ser.D. sembrerebbe segnata dalla cosiddetta "doppia dipendenza", ossia dipendenza nei confronti del partner e dipendenza nei confronti della sostanza (Malagoli Togliatti; Mazzoni, 1993) con una vera e propria mascolinizzazione dei propri stili di vita (Stocco et al., 2000). In altri termini, le donne tossicodipendenti assumerebbero una maschera maschile (male mask), ossia tratti caratteriali, a causa della condizione di addiction, tipicamente maschili come l'aggressività, la scarsa cura di sé, gli attacchi di panico al fine di garantirsi maggiori chance di sopravvivenza all'interno della subcultura del consumo.

Quanto deriverebbe da aspetti personali e patologie della personalità, I'indagine di Stocco e collaboratori non sembra approfondire più di tanto, lasciando aperta la questione del condizionamento dettato dalla rappresentazione sociale in termini di significati relativi al femminile e al maschile.

Il mondo delle sostanze, infatti, non sembra avulso da quei meccanismi materiali o simbolici che definiscono la dimensione del genere.

Provando a riflettere sul dato suggerito dall'OEDT a proposito dell'alcol, della cocaina, dell'ecstasy, ossia dove le differenze numeriche tra consumatori uomini e donne si assottigliano, la teoria della maschera maschile sembra perdere credibilità, in quanto I'utilizzo ricreazionale sembrerebbe vivificare i cliché identitari femminili in termini di sensualizzazione e sessualizzazione dell'ambiente del consumo ricreazionale.

Sia gli studi di Sheila Henderson (1997) che quelli di K. Williams (1998), spingerebbero a credere, infatti, che la sostanza è utilizzata dalle frequentatrici delle scena techno dance inglese (Ecstasy) e dei College dell'Est Coast (alcol) al fine di provocare condizioni di disinibizione empatogena senza mai sconfinare nelI'eccesso disconfermante, sotto il profilo dell'immagine di femminilità, della mancanza di controllo.

\section{Le donne e il "piacere" alcolico}

Solo di recente il bere delle donne è diventato un comportamento socialmente accettato, se pur con la pervicacia di alcune remore.

Da sempre il bere alcolici è stata considerata una pratica al maschile, sia in termini di consumi di qualità e di testimonianza di forza e virilità (Guarino, 2010) sia in termini di consumo problematico.

Sono scarsissime le testimonianze nella storia relative al bere femminile e consuetamente riferite alla perdizione e al malaffare.

La socializzazione ai ruoli che la società attuale propone, pur presentando ancora avamposti di strenua difesa delle differenze su molti campi, ha registrato un'indubbia revisione in termini di relazione e sessualità.

Si assiste innegabilmente ad una sua presa di distanza dalla tipizzazione tradizionale dei ruoli sociali, a vantaggio di una sorta di più attenuata differenziazione forte del pronunciarsi di alcuni simbolismi e pratiche.

I tatuaggi, i piercing, un certo tipo di abbigliamento, sembrano voler annullare la distinzione tra i sessi biologici pur continuando su altri piani a mantenere antiche e irrisolte rigidità.

Il dato relativo all'anagrafe sembra parlare di una tendenza che coinvolge le donne più giovani, sebbene questo aspetto non limita una sorta di sdoganamento delle stesse pratiche anche tra le generazioni precedenti.

Significazioni attribuibili al bere, modalità di accesso, modi di bere hanno minimizzato e normalizzando, la differenza di genere.

Se gli uomini continuano a bere di più, se in numero comunque maggiore sono registrati i pazienti di sesso maschile presso i Servizi della cura, questo non vuol dire che le donne non utilizzino alcol.

Si tratta di un bere leggero, ristretto entro i confini del fine settimana, non legato alla mascolinizzazione ma più ancorato alla voglia di rielaborare le distanze tra i sessi e le rappresentazioni sociali ad esse collegate.

Sembrerebbe che non è per sentirsi uomini che le donne bevono ma per affermare la propria femminilità non più da leggersi in chiave di fragilità.

La forza non è ad appannaggio dell'uomo, la donna che beve e sostiene la pratica del bere e, nei casi estremi del binge drinking I'ubriachezza, non intende affermare una sorta di mascolinità.

La donna che nel fine settimana utilizza sostanze stupefacenti per concedersi sballo, divertimento e sessualità, intende suggerire un'uguaglianza partendo da ciò da cui è stata tenuta più lontana per secoli: la trasgressione e il piacere.

Perché questo accada, però, è necessario affermare la liceità del comportamento, definirne una pratica mirata, provando a mantenerne il controllo e soprattutto ad applicarne il dissenso lì dove, come nel caso della maternità, la donna sembra riaffermare la necessità della sicurezza.

Quanto questa capacità di gestire il consumo in chiave ricreativa possa durare non è dato sapere al momento.

Se guardiamo ai dati ci rendiamo conto che la dipendenza femminile riguarda soprattutto alcol, psicofarmaci, cibo, nicotina e più di recente gioco.

Non dovrebbe essere difficile concludere che la donna si orienta al mondo della legalità o, comunque, della più facile accettazione sociale.

Nel caso di sostanze illegali il dato al femminile è ancora nel sommerso.

Per ciò che riguarda l'alcol è sotto gli occhi di tutti una sorta di sdoganamento dell'uso problematico e della dipendenza per le donne, sebbene le stesse siano ancora pochissime nei Servizi pubblici. Diverso è il dato fornito da Alcolisti Anonimi dove le donne sono tra il 30 e il $40 \%$.

Ci sarebbe da chiedersi quanto i Ser.D. e più ancora le Comunità terapeutiche dove i dati relativi alle presenze e alla ritenzione 
scendono ulteriormente, siano in grado di offrire "accoglienza" e cura alle donne. Quanto sarebbero in grado di rispondere ad una domanda non pronunciata, o percepita ancora come non pronunciabile dalla donna, di ascolto e parola rispetto all'uso/dipendenza?

\section{Conclusioni}

Nonostante secondo l'Istat continui a permanere una differenza tra il numero di assuntori uomini e quello relative alle donne, anche e soprattutto in termini di dipendenza, non si può disconoscere che già le conseguenze dell'uso problematico si fanno sentire più pesantemente tra le donne, sia per una questione di massa corporea, sia per una più ridotta capacità di metabolizzazione in termini epatici, cardiovascolari e psichiatrici.

È proprio in ragione di queste differenze di genere che uomini e donne potrebbero presentare delle differenze anche in chiave di efficacia dei trattamenti.

Dai risultati di una ricerca condotta dall'Università di Cagliari e di Sassari, pubblicati nei primi mesi del 2016 sulla Rivista European Addiction Research si evince che per molte molecole usate nel trattamento della dipendenza e della sindrome di astinenza (disulfiram, acamprosato, naltrexone, nalmefene, gamma-idrossibutirrato o GHB) I'efficacia non è stata adeguatamente verificata su un campione femminile.

Secondo Roberta Agabio del Dipartimento di Scienze biomediche dell'Università di Cagliari, sono stati analizzati 92 studi clinici per un totale di 15 mila partecipanti di cui uno soltanto condotto solo su donne a fronte di ben 19 solo su uomini.

Negli altri, le donne erano presenti in misura variabile, da un minimo dell' $1,2 \%$ ad un massimo del 31,8\% (Agabio et al., 2016).

Sembrerebbe di poter concludere che nei Servizi per le Dipendenze, le donne sono curate con farmaci la cui efficacia è verificata soprattutto sugli uomini.

Forse sarebbe il caso di promuovere studi in grado di monitorare la popolazione femminile nelle sue diverse fasce d'età.

Forse sarebbe il caso di riflettere anche su quanto tutti gli altri trattamenti proposti siano in grado di rispondere a più specifiche domande di aiuto.

Ci sembra di poter concludere che le donne nell'uso e paradossalmente nelle dipendenze continuano ad essere ufficialmente "invisibili" sebbene gli stessi non siano prerogative del genere maschile.

Sarebbe opportuno, dunque, rivedere gli strumenti della ricerca e più ancora la possibilità di leggere dati, cercando di addivenire a conclusioni che non siano solo somme e considerazioni meramente numeriche.

Se qualcosa non si vede, non è detto che non esista.

Magari si potrebbe provare a cambiare le lenti con cui guardare. A tal proposito, non si può non suggerire una riflessione anche sulla possibilità di pensare a Servizi più "femminili", rinnovando i legami con il profondo, il reale, la vita sociale e psichica. Considerato, poi, che la presenza femminile appare più frequente per ciò che attiene a consumi o comportamenti considerati dal punto di vista giuridico leciti (gioco d'azzardo, alcol, nicotina, psicofarmaci), riuscire a sdoganare l'idea di luogo della cura più che della semplice e riduttiva risposta a comportamenti "devianti", e per la maggior parte della gente devianti sotto il profilo penale, aiuterebbe a rendere la domanda di aiuto della donna consumatrice e dipendente più intercettabile e più realisticamente disponibile alla presa in carico e alla cura.

È mito del nostro tempo quello di bastare a se stessi, di negare il vincolo, il legame, forse anche il debito nei confronti dell'Altro.
Rivedere l'autotrofismo che esclude, che si soddisfa di sé, è ciò verso cui l'assenza della donna nei Servizi delle dipendenze dovrebbe indurre, focalizzando I'intervento degli operatori sulla possibilità di trascendere la dimensione anonima, predefinita e protocollare delle cure così come comunemente vengono offerte.

La particolarità dell'intervento terapeutico, l'individualità della domanda, la centralità della persona sono anche e soprattutto una cura non anonima, particolare, capace di parola e ascolto. Nell'esperienza dello specchio narcisistico, altro mito del nostro tempo, non può esserci riconoscimento ma solo immagine posta di fronte a se stessa.

Per questo i Servizi per le dipendenze, cercando di fronteggiare le conseguenze di uno stigma sociale particolarmente presente e pressante nei confronti delle donne, dovrebbero sapersi rendere custodi di parole, restituendo ai pazienti il senso delle stesse e dei silenzi.

Questo non vuol dire interpretare o assegnare significati ma lavorare affinché la persona avvii un viaggio/percorso di ricerca e riconoscimento di sé e, forse, tutto ciò per ciò che attiene all'universo femminile significa provare nuovi approcci.

Una nuova sfida per i Servizi che non si può più disattendere.

\section{Bibliografia}

Report SPS (School Population Survey) Puglia, Anno 2015. Indagine Sul Consumo di sostanze Psicotrope negli Studenti delle Scuole Secondarie di secondo grado. A cura di: Dipartimento della salute, del Benessere Sociale e dello Sport per Tutti Regione Puglia; Osservatorio Epidemiologico Regione Puglia; Ufficio Scolastico Regionale.

RAPPORTI ISTISAN 16/4 Anno 2016 "Epidemiologia e Monitoraggio alcolcorrelato in Italia e nelle Regioni". A cura di E. Scafato, C Gandin, L. Di Pasquale, L. Galluzzo, S. Martire, S. Ghirini per il gruppo di Lavoro CSDA (Centro Servizi Documentazione Alcol).

Abraham G. (2002), I mille volti del piacere, Mondadori, Milano.

Agabio R., Pani P.P., Preti A., Gessa G.L., Franconi F. (2016), "Efficacy of Medications Approved for the Treatment of Alcohol Dependence and Alcohol Withdrawal Syndrome in Female Patients: A Descriptive Review", European Addiction Research, 22: 1-16 (DOI: 10.1159/000433579).

Cipolla C., Martoni M. (a cura di) (2009), Droghe nella notte. Una ricerca empirica sulla costa romagnola, FrancoAngeli, Milano.

Cipolla C. (a cura di) (2013), Droghe al femminile, FrancoAngeli, Milano.

Dionigi A., Pavarin R.M. (2010), Sballo, Erickson Educazione, Trento.

EMCDDA (2006), Selected Issues. A gender perspective on drug use and responding to drug problems, disponibile al link www.emcdda. europa.eu/attachements.cfm/att_37291_EN_sel2006_2-en.pdf.

Formica A., Giuliodoro S. (2009), "Tossicodipendenza femminile e maternità", Salute e Prevenzione, 52: 33-42.

Guarino F. (2010), Alcol e stile giovane. Un'interpretazione sociologica, FrancoAngeli, Milano.

Henderson S. (1997), Ecstasy: Case Unsolved, Pandora, Londra.

Linden J.D. (2012), La bussola del piacere, Codice edizioni, Torino.

Malagoli Togliatti M., Mazzoni S. (a cura di) (1993), Maternità e tossicodipendenza, Giuffrè, Milano.

Parker H., Aldridge J. (1998), Illegal leisure. The normalization of Adolescent Recreational Drug Use, Routledge, London.

Parker H., Williams L., Aldridge J. (2002), "The Normalization of 'Sensible' Recreational Drug Use", Sociology, vol. 36, n. 4: 941-964.

Stocco P., Llacer J.L., De Fazio L., Calafat A., Mendes F. (2000), Women Drug Abuse in Europe: Gender Identity, IREFREA, Palma de Mallorca.

Wallace M., Eysenck S., Eysenck H.J. (1978), "Sensation seeking in England and America: Cross-cultural, age, and sex comparisons", Journal of Consulting and Clinical Psychology, 46: 139-149.

Williams K.M. (1998), Learning Limist. College Women, Drugs and Relationships, Bergin \& Garvey, Westport.

Zuckerman M. (1983), Biological Bases of Sensation Seeking, Impulsivity and Anxiety, Erlbaum, Hillsdale, N.J. 